\title{
Selective Down-Regulation of Aquaporin-1 in Salivary Glands in Primary Sjögren's Syndrome
}

\author{
Dimitra Beroukas, Jenny Hiscock, Bren J. Gannon, Roland Jonsson, \\ Tom P. Gordon, and Sally A. Waterman \\ Departments of Immunology, Allergy, and Arthritis (DB, JH, TG, SW) and Anatomy and Histology (BG), Flinders \\ Medical Centre and Flinders University, Bedford Park, Australia; and Broegelmann Research Laboratory (RJ), \\ University of Bergen, Bergen, Norway
}

\begin{abstract}
SUMMARY: Salivary and lacrimal gland secretions are reduced in primary Sjögren's syndrome (pSS). Aquaporins (AQPS) are involved in transmembrane water transport, and different isoforms show specific cellular and subcellular distributions in salivary and lacrimal glands. Changes in expression of AQP molecules have therefore been suggested to contribute to the glandular dysfunction in pSS. AQP-5 is present in the apical membrane of acinar cells, where it mediates fluid outflow; however, we have recently shown that its expression is not altered in pSS. We therefore studied whether expression of other isoforms of AQP would be altered in pSS. Using high-resolution confocal microscopy, we determined the distribution of AQP-1 and AQP-3 in labial salivary gland biopsies from 11 patients with pSS and 9 healthy controls. AQP-1 is present in myoepithelial cells surrounding acini, and its expression in these cells was decreased by $38 \%$ in pSS glands. By contrast, expression of AQP-1 in endothelial cells of nonfenestrated capillaries was not altered in pSS. AQP-3 was expressed in the basolateral membrane of acinar epithelial cells, and its expression was not altered in disease. We therefore conclude that AQP-1 expression in myoepithelial cells is selectively down-regulated in pSS and that myoepithelial cell dysfunction may play a crucial role in the pathology of this disease. (Lab Invest 2002, 82:1547-1552).
\end{abstract}

$P$ rimary Sjögren's syndrome (pSS) is an autoimmune disease of unknown cause and is characterized clinically by dry eyes and mouth (sicca syndrome), indicating a reduction in fluid secretion from lacrimal and salivary glands. Recent studies suggest that these symptoms are due to functional inhibition of lacrimal and salivary glands rather than to infiltration and destruction of the glands by T-lymphocytes, because there is a poor correlation between degree of glandular destruction and degree of dysfunction (Humphreys-Beher et al, 1999). Functional inhibition of these exocrine glands could reflect inhibition of neuroeffector transmission or a defect downstream in the postjunctional intracellular second messenger systems required for stimulus-secretion coupling.

Aquaporins (AQPs) are water channel proteins that provide a pathway for osmotic water flow across cell membranes (Nielsen et al, 1997), including across salivary epithelial cells. Much attention has recently been focused on the role of AQPs in pSS, and changes in their expression have been proposed to

\section{DOI: 10.1097/01.LAB.0000038502.42845.9E}

Received July 18, 2002.

This work was funded by grants from the National Health and Medical Research Council of Australia (980180 to SAW). SAW is a National Health and Medical Research Council of Australia R.D. Wright Fellow (987761).

Address reprint requests to: Dr. Sally A. Waterman, Department of Immunology, Allergy and Arthritis, School of Medicine, Flinders University, GPO Box 2100, Adelaide SA 5001 Australia. E-mail: Sally.Waterman@flinders.edu.au underlie the sicca symptoms (Steinfeld et al, 2001). In human salivary glands, $A Q P-5$ is restricted to the apical membrane of acinar cells, where it allows fluid outflow into the acinar lumen. In mice mutant for the AQP-5 channel, salivary gland secretion is reduced (Ma et al, 1999), and it is tempting to speculate that altered distribution or function of this molecule in pSS could explain the sicca symptoms. However, we recently demonstrated using high-resolution confocal microscopy and quantitative image analysis that expression of AQP-5 is unchanged in pSS (Beroukas et al, 2001); other groups have reported changes in AQP-5 expression in pSS glands (Steinfeld et al, 2001; Tsubota et al, 2001), although these studies did not use high-resolution microscopic techniques or antigen retrieval methods.

Normal functioning of the salivary gland must depend not only on water outflow through AQP-5 channels into the acinar lumen but also on water flow into the acinar epithelial cells and perhaps also the myoepithelial cells that surround them. AQP isoforms are differentially expressed in different subcellular compartments in salivary glands: AQP-3 has been reported to be localized to the basolateral surface of acinar epithelial cells, where it presumably allows water inflow into these cells, and AQP-1 is expressed in myoepithelial cells (Gresz et al, 2001). We therefore studied the distribution of AQP-1 and AQP-3 isoforms in control and pSS labial salivary gland (LSG) biopsies using high-resolution confocal microscopy and quantitative image analysis. 


\section{Results}

Indirect immunofluorescence detection showed that the distribution of AQP-1 labeling was confined to the basal region of acini and intercalated ducts as shown previously, probably in myoepithelial cells (Gresz et al, 2001). AQP-1 labeling patterns were similar in both control and pSS glands (Fig. 1, A to C); however, the labeling was less intense in glands from each of the patients with pSS. AQP-1 labeling was also present in endothelial cells of nonfenestrated capillaries; however, the intensity of this labeling did not alter in pSS (Fig. 1, B and C). In the apical and lateral membranes of acinar cells (Fig. 1, A to C), in striated ducts, and in infiltrating lymphocytes, AQP-1 labeling was absent. All labeling was abrogated by preabsorption with 100 $\mu \mathrm{g} / \mathrm{ml}$ of the human AQP-1 peptide (Fig. 1D) but not by unrelated peptide (AQP-5; not shown). Using highresolution confocal microscopy, we confirmed the basal labeling of AQP-1 in acini and intercalated ducts.

By contrast, AQP-3 labeling was present in the basolateral membrane of the acinar cells, as described previously, but not in intercalated or striated ducts (Gresz et al, 2001). This pattern was similar in glands from healthy control subjects and patients with
pSS, and the intensity of the labeling seemed similar between control subjects and patients with pSS.

To determine precisely the subcellular localization of AQP-1, we performed double-labeling immunofluorescence. Labeling myoepithelial cells with an $\alpha$-smooth muscle actin antibody (Fig. 2) indicated that the AQP-1 labeling is present in the myoepithelial cell membrane adjacent to the acinar epithelial cells and to a lesser extent in the membrane farthest from the acinus. Although AQP-1 labeling was decreased in the myoepithelial cells of pSS glands, $\alpha$-smooth muscle actin labeling remained unchanged. Double-labeling for the M3-muscarinic receptor (M3R), which we have previously demonstrated is present on myoepithelial cells (Beroukas et al, 2002), confirmed that AQP-1 is on the myoepithelial cell membrane nearest the acinus (data not shown).

High-resolution confocal microscopy was used to measure quantitatively the differences in AQP-1 and AQP-3 labeling in pSS and control LSG biopsies. Quantification of fluorescence intensity of myoepithelial AQP-1 labeling of 10 acini per 5 control and 6 pSS glands on confocal microscopic images showed a highly significant decrease in pixel intensity in the same patients with pSS who had previously shown no
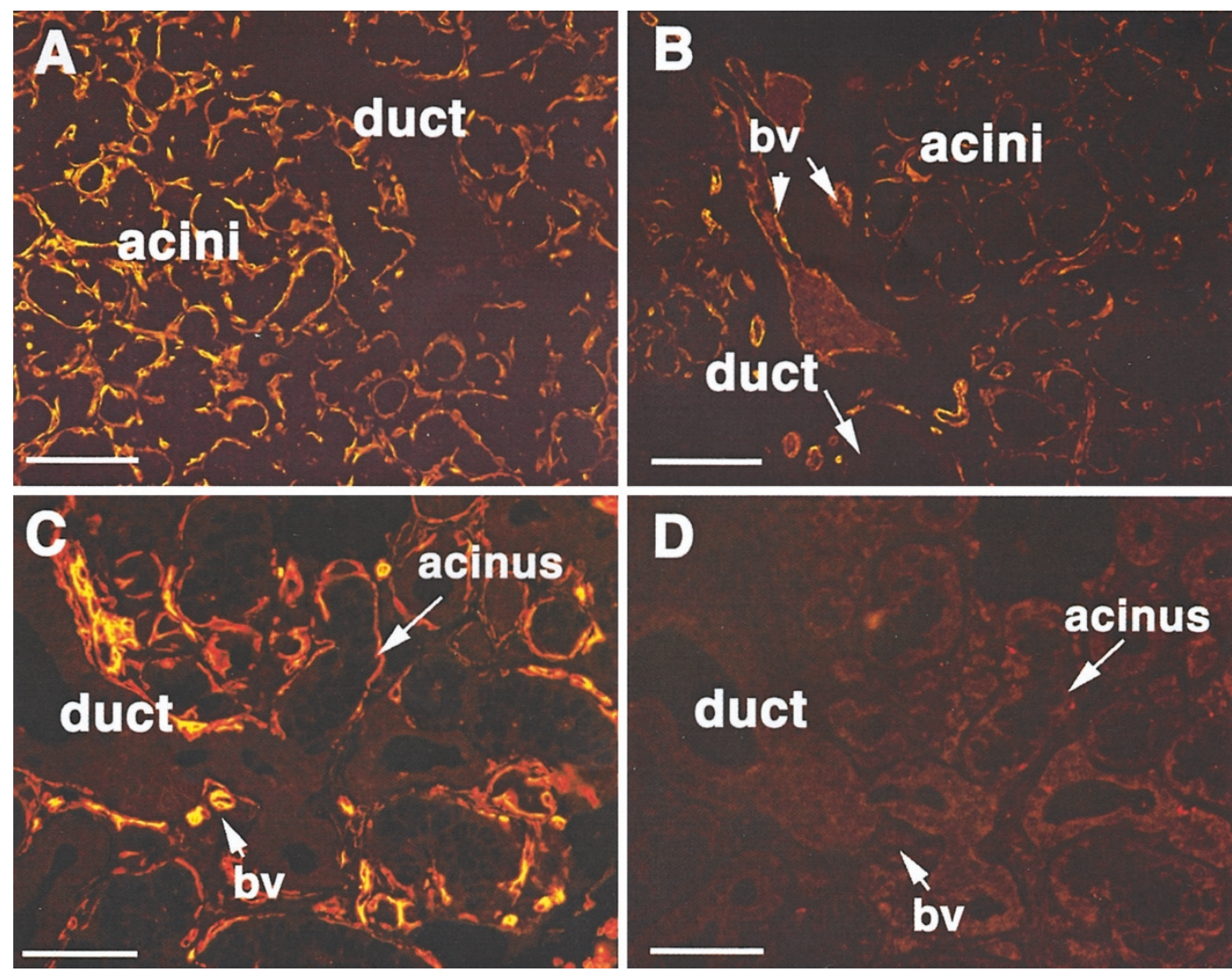

Figure 1.

Immunolocalization of aquaporin (AQP)-1 in labial salivary gland biopsies. Sections of biopsy samples were from normal control subjects (A, C, and D) and a patient with primary Sjögren's syndrome (pSS; B). Sections were labeled with an affinity-purified polyclonal antibody to human AQP-1 and visualized using fluorescent-labeled secondary antibody with conventional fluorescence microscopy ( $A$ and $B$ ) or confocal microscopy ( $C$ and $D$ ). AQP-1 was detected in the basal aspect of acini in both control and pSS glands, but the intensity of labeling was greatly decreased in pSS glands. AQP-1 labeling also occurs in blood vessels (bv; B and C) and intercalated ducts (C). Labeling was blocked by preabsorption with the human AQP-1 peptide (D). Scale bars $=100 \mu \mathrm{m}(\mathrm{A}$ and B) and $50 \mu \mathrm{m}(C$ and D). 


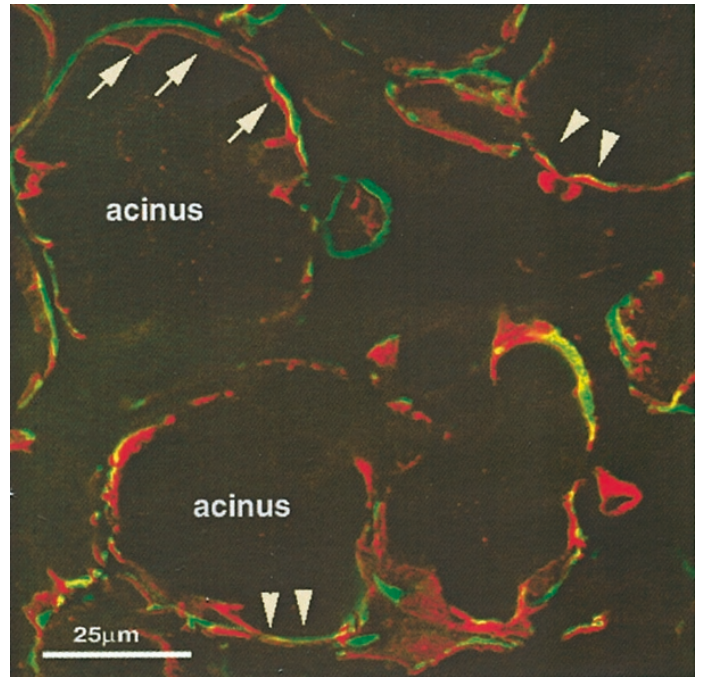

Figure 2.

Co-localization of AQP-1 with $\alpha$-smooth muscle actin in labial salivary gland biopsies. Actin (green) is used to label myoepithelial cells that surround the secretory acini and intercalated ducts. AQP-1 labeling (red) appears predominantly as continuous membrane labeling luminal to the actin in the myoepithelial cell (arrows). AQP-1 also occurs on the myoepithelial cell membrane farthest from the acinus (arrowheads).

difference in AQP-5 labeling (Beroukas et al, 2001) and increased M3R expression (Beroukas et al, 2002) (Fig. $3 A)$. Investigators who viewed sections were masked

A

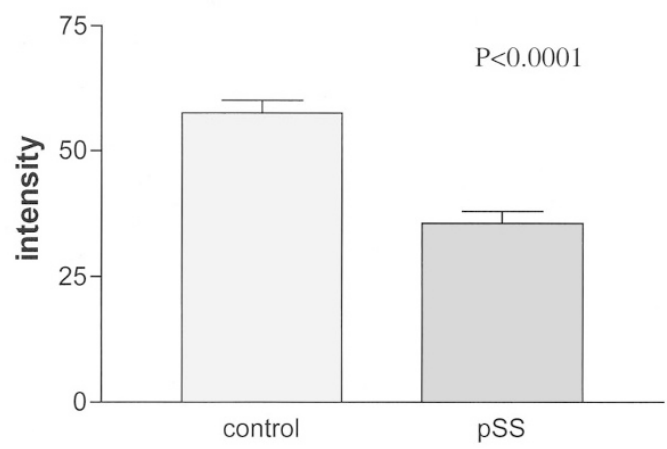

B

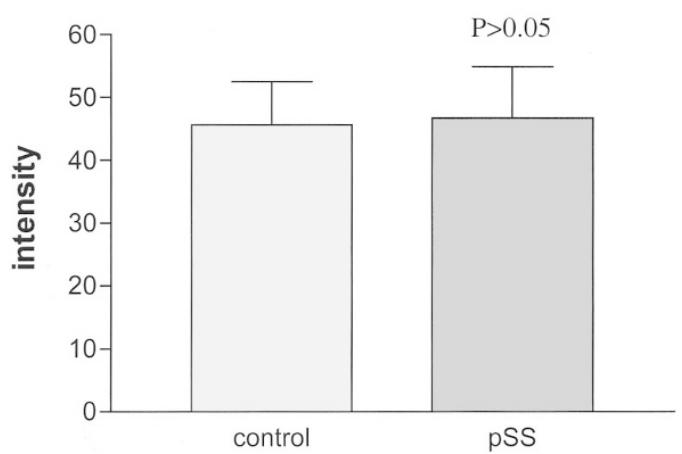

Figure 3.

Fluorescent intensity of labeling for AQP-1 (A) and AQP-3 (B) in myoepithelial cells and salivary acinar cells in labial salivary gland biopsies from healthy controls and patients with pSS. to group, and all images were taken at the same settings on a Bio-Rad 1024 laser scanning confocal system (Hemel Hempstead, United Kingdom). Using identical sampling techniques, we found no significant difference in the intensity of AQP-3 labeling in the basolateral membrane of acinar epithelial cells in control versus pSS patient glands (Fig. 3B).

\section{Discussion}

Our studies suggest that the capacity for water flow across the membrane(s) of myoepithelial cells may be altered in pSS, because AQP-1 expression in these cells is selectively decreased. However, AQP-3 expression in the basolateral membrane and AQP-5 expression in the apical membrane of the same population of acinar epithelial cells are unaltered. If one assumes that the AQP molecules detected immunohistochemically are functional, then water flow into and out of the acinus is unlikely to be rate-limiting in pSS.

\section{Role of AQP-1 and Myoepithelial Cells in Exocrine Gland Function}

Myoepithelial cells have tapering processes that embrace the acini and may assist in salivary flow and provide structural support in the face of increased intraluminal pressure during salivary secretion (Sato et al, 1979). Acetylcholine acts on M3R on myoepithelial cells (Beroukas et al, 2002) and causes their contraction (Satoh et al, 1994). The presence of M3R (Beroukas et al, 2002) and AQP-1 (Gresz et al, 1999, 2001; present study) on salivary gland myoepithelial cells also raises the interesting possibility that some of the effects of acetylcholine on secretion may be mediated indirectly through an action on the myoepithelial cells rather than through a direct effect of acetylcholine on the acinar cells. Because myoepithelial cells embrace acini, they could constitute a barrier to water flow into the acinar cells; the presence of AQP-1 in myoepithelial cells might ensure that water flow into the basal aspect of the acinar epithelial cells is not restricted (Gresz et al, 2001).

Activation of M3R results in inositol trisphosphate synthesis and subsequent increase in cytoplasmic calcium concentration via calcium release from inositol trisphosphate-sensitive intracellular stores. Increased cytoplasmic calcium activates $\mathrm{Ca}^{2+}$ dependent $\mathrm{K}^{+}$and $\mathrm{Cl}^{-}$channels and, by analogy with rat parotid salivary gland (Ishikawa et al, 1998), may cause a translocation of AQP-1 from intracellular vesicles to the membrane, thereby allowing water inflow and outflow through AQP-1 channels of myoepithelial cells. This mechanism is likely to be important in the rapid control of myoepithelial cell volume, which may be required to enable these cells to provide structural support during salivary secretion (Sato et al, 1979). The significant down-regulation of AQP-1 expression in myoepithelial cells (present study) suggests that these cells do not function normally in pSS glands. 


\section{Mechanisms that Lead to AQP-1 Down-Regulation}

Two principal mechanisms are likely to contribute to down-regulation of AQP-1 in pSS myoepithelial cells. First, studies on lung epithelium and parotid glands have demonstrated that cytokines can up- and downregulate the expression of AQP molecules (Smith et al, 1999; Towne et al, 2001), and cytokines are known to be produced in increased quantities by acinar cells and lymphocytes in pSS glands (Boumba et al, 1995; Fox et al, 1994; Grisius et al, 1997). Second, AQP-1 expression may be altered as a consequence of functional inhibition of cholinergic neurotransmission. Translocation of AQP-5 molecules to the apical membrane of rat parotid acinar cells is dependent on M3R stimulation (Ishikawa et al, 1998). If the normal expression of AQP-1 in myoepithelial cells is similarly dependent on intact M3R-mediated neurotransmission, then the decreased expression of AQP-1 may be a secondary consequence of the partial block of M3R-mediated neurotransmission in pSS glands (Waterman et al, 2000). It is interesting that in regenerating denervated skeletal muscle, AQP-4 expression is reduced, suggesting a role for nerves in the expression of skeletal muscle AQP (Jimi et al, 2000).

\section{Mechanisms of Exocrine Gland Dysfunction in pSS}

There is evidence that four mechanisms may contribute to salivary and lacrimal gland dysfunction in pSS, before lymphocytic destruction of the glands. First, anti-M3R antibodies are likely to block cholinergic neurotransmission to the glands (Waterman et al, 2000). Second, water flow across myoepithelial cells may be restricted as a result of AQP-1 downregulation; myoepithelial cells would then provide a barrier to water supply to acinar cells. Third, the supporting role of myoepithelial cells during salivary secretion is likely to be impaired as a result of downregulation of AQP-1 and impaired cell volume regulation (see above), and this may have secondary consequences on salivary and lacrimal outflow. Last, even if M3Rs are activated, second messenger systems are down-regulated in pSS (Dawson et al, 2001).

\section{Role of AQP-1 in Myoepithelial Cell Function}

Our observation that AQP-1 is expressed on the cell membranes of myoepithelial cells that surround the acini of salivary glands has intriguing parallels in the observations of AQP expression in the cell membranes of other excitable contractile cells, including those of smooth muscle cells (Gannon et al, 2000; Shanahan et al, 1999), cardiac muscle cells (Page et al, 1998), and the sarcolemma of fast-twitch skeletal muscle fiber (Frigeri et al, 1998). AQP-1 has been shown to mediate accelerated smooth muscle volume changes in response to altered external osmolarity (Shanahan et al, 1999), suggesting that variation in cell volume mediated by AQP-1 may contribute to contraction and/or variation in tone of vascular smooth muscle (Shanahan et al, 1999). Similarly, we propose that AQP-1 allows rapid changes in myoepithelial cell volume; increased myoepithelial cell volume would constrict the lumen of the salivary acinus and facilitate saliva flow. Physiological studies are necessary to test this hypothesis.

Using high-resolution confocal microscopy with image analysis (Beroukas et al, 2002), we have been unable to confirm earlier reports that AQP-5 expression in the apical membrane of acinar cells is decreased or altered in pSS glands (Steinfeld et al, 2001; Tsubota et al, 2001). Furthermore, AQP-3 expression in the basal membrane of acinar cells also is not altered in pSS glands (present study). We therefore propose that the water inflow and outflow capacity of the acinar epithelial cells themselves could potentially be normal in pSS glands and is decreased only as a consequence of the four mechanisms described above.

In conclusion, AQP-1 expression in myoepithelial cells is selectively down-regulated in pSS. Furthermore, the myoepithelial cell may play a more important role in salivary secretion than has previously been recognized.

\section{Materials and Methods}

\section{Human Tissues}

Salivary gland tissue was obtained by labial biopsy from nine patients (mean age $51 \pm 12$ years) who fulfilled the European consensus criteria for the diagnosis of pSS, including seropositivity for anti-Ro/La antibodies, unstimulated salivary flow $<1.5 \mathrm{ml}$ per 15 minutes, and LSG biopsy focus scores of $>1$ per 4 $\mathrm{mm}^{2}$. Disease duration ranged from 1 to 22 years. Biopsy tissues were obtained from 11 healthy control subjects (mean age $41 \pm 9$ years; unstimulated salivary flow of $4.7 \pm 2 \mathrm{ml}$ per 15 minutes). Control LSG tissues were histologically normal, with no foci of lymphocytes. All patients had been studied previously for LSG M3R and AQP-5 expression (Beroukas et al, 2001, 2002).

\section{Immunohistochemical Localization on Paraffin-Embedded Tissue}

Indirect immunofluorescence was used to detect the expression of the AQP-1 and AQP-3 in paraffinembedded LSG. A random $4 \mu \mathrm{M}$-thick section was cut for each case and dried onto histogrip-coated slides overnight at $57^{\circ} \mathrm{C}$. The sections were deparaffinized, microwaved for 10 minutes (Toshiba microwave at $1000 \mathrm{~W})$ using the microwave antigen-retrieval method of Shi et al (1991) in a citrate buffer ( $\mathrm{pH}$ 6.0), and blocked with neat Protein Block Serum-Free (DAKO Corporation, Carpinteria, California). Sections were incubated with a previously characterized affinity-purified rabbit antibody raised against the 19amino acid sequence (aa 251-269) of the $\mathrm{COOH}$ terminus of human AQP-1 (Alpha Diagnostic, San Antonio, Texas) or against a 15-amino acid $\mathrm{COOH}-$ terminus sequence of rat AQP-3 (Alpha Diagnostic). The rat AQP-3 sequence shares $88 \%$ homology with human AQP-3. Sections were then washed with $0.1 \%$ 
Tween 20 in Tris-buffered saline (pH 7.4), and the antibody binding was visualized using a donkey antirabbit IgG conjugated to indocarbocyanin fluorophore (Cy-3, Jackson Immunoresearch, West Grove, Pennsylvania; 1:100 dilution for 2 hours at room temperature), washed, and mounted in 1,4 Diazabicyclo-(222)octane, phospho-glycerol. For dual-labeling studies, sections were incubated with AQP-1 antibody together with a mouse monoclonal antibody raised against the human $\alpha$-smooth muscle actin (MAB-003, 1:100; Maxim Biotech, San Francisco, California) or a goat polyclonal raised against human M3-muscarinic receptor (M3R-C20, 1:50; Santa Cruz Biochemicals, Santa Cruz, California). The smooth muscle actin antibody was visualized with the use of a donkey anti-mouse FITC (1:100; Jackson), and the M3R antibody was visualized with the use of donkey anti-goat Cy-3 (1:100 dilution; Jackson). Consecutive sections from each tissue block were stained with Mayer's hematoxylin and eosin for histological examination. Sections were viewed using a Leica DMLB-fluorescent microscope, and images were captured using a Leica DC200 digital camera (Leica, Sydney, Australia). Digital images were processed to match contrast and brightness and assembled into panels with Adobe Photoshop v5.5 (Adobe Systems, Mountain View, California).

\section{Controls}

The specificity of the primary antibody staining was determined by (a) omission of primary antibody from incubations, (b) preabsorption of the primary antibody with $100 \mu \mathrm{g} / \mathrm{ml}$ of the 19-amino acid immunizing peptide overnight at $4^{\circ} \mathrm{C}$ before section labeling, and (c) preabsorption as in (b) but using unrelated peptide AQP-5 (Alpha Diagnostic).

\section{Confocal Microscopy}

Random sections were viewed in an Olympus AX70 microscope attached to a Bio-Rad 1024 scanning confocal system equipped with a helium neon (543 $\mathrm{nm}$ ) and argon (488 $\mathrm{nm}$ ) laser for excitation and detection of CY3 and FITC, respectively. The confocal settings for capturing images were obtained from the sample with the brightest fluorescence; the samples had been coded to ensure unbiased collection of data. Images of acini were collected according to the following procedure. Confocal settings were optimized with regard to saturation and were laser power $1 \%$, iris 2.0, photomultiplier gain 915 , and $\times 100$ objective lens with software zoom of 3.5. Ten fields $(31.2 \mu \mathrm{m} \times 31.2$ $\mu \mathrm{m}$ in size) were captured from each sample. The position of the first field was chosen at random, and subsequent fields were chosen systematically in a $100-\mu \mathrm{m}$ grid pattern and two regions of gland were sampled, five fields from each area. The grid interval of $100 \mu \mathrm{m}$ allowed each new field to sample a new acinus.

\section{Quantitative Measurement of Fluorescent Intensity}

The average intensity of the fluorescent labeling of AQP-1 or AQP-3 located in the membrane of the myoepithelial or acinar epithelial cells was determined using the Bio-Rad Lasersharp image analysis program (Bio-Rad, Hemel Hempstead, United Kingdom). Two steps were taken to ensure that our quantitative measurement was unbiased: measurements were made by an investigator who was blinded to the nature of the coded LSG sample, and measurements were made systematically of 10 acini per sample, selected using a $100-\mu \mathrm{m}$ grid. The total regions of AQP-1 or AQP-3 labeling were circumscribed with a computer mouse in 6 to 10 outlines per field, and the intensity of fluorescence was measured as pixel intensity. Three additional areas were outlined in regions without fluorescent labeling, for determining the average intensity of the background. These background measurements were averaged and then subtracted from each measurement of AQP-1 or AQP-3 fluorescence to provide a measure of absolute fluorescence intensity. Values for the fluorescence intensity were averaged per field, giving a total of 60 to 80 measurements of membrane fluorescence per sample (patient or control).

\section{Statistical Analysis}

Results were expressed as mean fluorescence intensity \pm SEM. Differences in fluorescence intensity between healthy control and pSS samples were analyzed using Mann-Whitney $U$ tests. $p<0.05$ was considered significant.

\section{Acknowledgements}

We thank Dr. David Wilson (Institute of Medical and Veterinary Science, Adelaide) for providing normal labial salivary gland biopsies and Ms. Dana Cavill for technical assistance.

\section{References}

Beroukas D, Goodfellow R, Hiscock J, Jonsson R, Gordon TP, and Waterman SA (2002). Upregulation of M3-muscarinic receptors in labial salivary gland acini in primary Sjögren's syndrome. Lab Invest 82:203-210.

Beroukas D, Hiscock J, Jonsson R, Waterman SA, and Gordon TP (2001). Subcellular distribution of aquaporin 5 in salivary glands in primary Sjogren's syndrome. Lancet 358: 1875-1876.

Boumba D, Skopouli FN, and Moutsopoulos HM (1995). Cytokine mRNA expression in the labial salivary gland tissues from patients with primary Sjogren's syndrome. Br J Rheumatol 34:326-333.

Dawson LJ, Field EA, Harmer AR, and Smith PM (2001). Acetylcholine-evoked calcium mobilization and ion channel activation in human labial gland acinar cells from patients with primary Sjogren's syndrome. Clin Exp Immunol 124: 480-485.

Fox RI, Kang HI, Ando D, Abrams J, and Pisa E (1994). Cytokine mRNA expression in salivary gland biopsies of Sjogren's syndrome. J Immunol 152:5532-5539. 
Frigeri A, Nicchia GP, Verbavatz JM, Valenti G, and Svelto M (1998). Expression of aquaporin-4 in fast-twitch fibers of mammalian skeletal muscle. J Clin Invest 102:695-703.

Gannon BJ, Warnes GM, Carati CJ, and Verco CJ (2000). Aquaporin-1 expression in visceral smooth muscle cells of female rat reproductive tract. J Smooth Muscle Res 36:155167.

Gresz V, Burghardt B, Ferguson CJ, Hurley PT, Takacs M, Nielsen S, Varga G, Zelles T, Case RM, and Steward MC (1999). Expression of aquaporin 1 (AQP1) water channels in human labial salivary glands. Arch Oral Biol 44(Suppl 1):S53S57.

Gresz V, Kwon TH, Hurley PT, Varga G, Zelles T, Nielsen S, Case RM, and Steward MC (2001). Identification and localization of aquaporin water channels in human salivary glands. Am J Physiol 281:G247-G254.

Grisius MM, Bermudez DK, and Fox PC (1997). Salivary and serum interleukin 6 in primary Sjogren's syndrome. J Rheumatol 24:1089-1091.

Humphreys-Beher MG, Brayer J, Yamachika S, Peck AB, and Jonsson R (1999). An alternative perspective to the immune response in autoimmune exocrinopathy: Induction of functional quiescence rather than destructive autoaggression. Scand J Immunol 49:7-10.

Ishikawa Y, Eguchi T, Skowronski MT, and Ishida H (1998). Acetylcholine acts on M3 muscarinic receptors and induces the translocation of aquaporin5 water channel via cytosolic $\mathrm{Ca} 2+$ elevation in rat parotid glands. Biochem Biophys Res Commun 245:835-840.

Jimi T, Wakayama Y, Murahashi M, Shibuya S, Inoue M, Hara $\mathrm{H}$, Matsuzaki Y, and Uemura N (2000). Aquaporin 4: Lack of mRNA expression in the rat regenerating muscle fiber under denervation. Neurosci Lett 291:93-96.

Ma T, Song Y, Gillespie A, Carlson EJ, Epstein CJ, and Verkman AS (1999). Defective secretion of saliva in transgenic mice lacking aquaporin-5 water channels. J Biol Chem 274:20071-20074.

Nielsen S, King LS, Christensen BM, and Agre P (1997). Aquaporins in complex tissues. II. Subcellular distribution in respiratory and glandular tissues of rat. Am J Physiol 273: C1549-C1561.
Page E, Winterfield J, Goings G, Bastawrous A, and UpshawEarley J (1998). Water channel proteins in rat cardiac myocyte caveolae: Osmolarity-dependent reversible internalization. Am J Physiol 274:H1988-H2000.

Sato K, Nishiyama A, and Kobayashi M (1979). Mechanical properties and functions of the myoepithelium in the eccrine sweat gland. Am J Physiol 237:C177-C184.

Satoh Y, Oomori Y, Ishikawa K, and Ono K (1994). Configuration of myoepithelial cells in various exocrine glands of guinea pigs. Anat Embryol (Berl) 189:227-236.

Shanahan CM, Connolly DL, Tyson KL, Cary NR, Osbourn JK, Agre P, and Weissberg PL (1999). Aquaporin-1 is expressed by vascular smooth muscle cells and mediates rapid water transport across vascular cell membranes. J Vasc Res 36:353-362.

Shi SR, Key ME, and Kalra KL (1991). Antigen retrieval in formalin-fixed, paraffin-embedded tissues: An enhancement method for immunohistochemical staining based on microwave oven heating of tissue sections. J Histochem Cytochem 39:741-748.

Smith JK, Siddiqui AA, Modica LA, Dykes R, Simmons C, Schmidt J, Krishnaswamy GA, and Berk SL (1999). Interferon-alpha upregulates gene expression of aquaporin-5 in human parotid glands. J Interferon Cytokine Res 19:929935.

Steinfeld S, Cogan E, King LS, Agre P, Kiss R, and Delporte C (2001). Abnormal distribution of aquaporin- 5 water channel protein in salivary glands from Sjogren's syndrome patients. Lab Invest 81:143-148.

Towne JE, Krane CM, Bachurski CJ, and Menon AG (2001). Tumor necrosis factor-alpha inhibits aquaporin 5 expression in mouse lung epithelial cells. J Biol Chem 276:18657-18664.

Tsubota K, Hirai S, King LS, Agre P, and Ishida N (2001). Defective cellular trafficking of lacrimal gland aquaporin-5 in Sjogren's syndrome. Lancet 357:688-689.

Waterman SA, Gordon TP, and Rischmueller M (2000). Inhibitory effects of muscarinic receptor autoantibodies on parasympathetic neurotransmission in Sjogren's syndrome. Arthritis Rheum 43:1647-1654. 